\title{
Benefits of screening cancer patients for distress still not demonstrated
}

\author{
J C Coyne $e^{*, 1}$ \\ ${ }^{1}$ Department of Psychiatry, Perelman School of Medicine, University of Pennsylvania, 3535 Market Street, Room 676, Philadelphia, \\ PA 19104, USA
}

Sir,

In the randomised controlled trial reported by Carlson et al (2012), screening cancer patients for distress was followed by one of the two different triages: computerised vs personalised. The authors concluded that screening for distress is 'a viable intervention that has the potential to decrease symptom burden up to 12 months post diagnosis.' It is not clear what is meant by 'viable' in this context, but this study provided no resolution of the more basic question of whether screening improves patient outcomes relative to the results achieved in routine care without screening.

When main effects and two- and three-way interactions presented in table 2 in the paper by Carlson et al (2012) are examined, it is apparent that changes in distress occurring with the passage of time $(P<0.0001)$ dwarfed changes attributable to a particular triage or the interaction between time and triage assignment, neither of which approached statistical significance. One explanation is simply that the study demonstrated a wellestablished trajectory of declining distress over time following a diagnosis of cancer, without screening and triage having altered this.

The trial failed to provide the comparison crucial to evaluating the benefits of screening in decreasing 'symptom burden up to 12 months post diagnosis.' Consistent with standards employed in evaluating screening in medicine more generally (Raffle and Gray, 2007), screening for distress would be judged efficacious if it were shown to improve patient outcomes beyond what would be achieved in routine care. Screening must be shown to improve upon patients having access to discussions with peers and professionals, as well as psychosocial services, but without screening being provided and without patients having to score above an a priori cut point for distress in order to have discussions or access services.

Carlson et al (2012) justified exclusion of a routine care comparison condition because previous work has already established the feasibility of screening in cancer settings, and the superiority of screening with triage to screening without triage
(Carlson et al, 2010), so it seemed somewhat unethical not to offer some form of triage.

It is worth examining whether Carlson et al (2010) yielded results so compelling that to offer routine care condition without screening would be 'somewhat unethical.' That previous trial compared three conditions: (a) screening for distress but withholding feedback to patients or clinicians; (b) screening generating a personalised report provided to patients and placed in an electronic medical record; and (c) patients receiving in addition to the report an offer to discuss any issues with staff, regardless of their level of distress. The first condition approximated a routine care condition. At 3 months, the groups did not differ on anxiety or depressive symptoms. However, the group in which patients were offered feedback and a chance to discuss psychosocial issues with staff, regardless of their distress score, demonstrated a small ( $<1$ point) but significant decrease in distress relative to patients receiving minimal screening or no access to screening results. Reflecting the marginal significance of these results, patient distress in neither of these groups was significantly different from the group for which screening results were shared but without an offer of discussion with staff. This trial hardly demonstrates the efficacy of screening and certainly does not support the argument that leaving patients in routine care is 'rather unethical.'

In hindsight, Carlson et al (2011) noted that group differences may have been attenuated by all patients, regardless of group assignment, meeting with a research assistant who provided them with literature and phone numbers of the psychosocial resources department, and informed them that they could contact the department and speak to someone about their concerns. Although quite plausible, this explanation suggests that simply providing patients with an opportunity for a minimal discussion with information and encouragement to seek services, regardless of level distress, might provide the benefits sought by implementing screening. Carlson et al (2012) does nothing to unseat this conclusion.

However, Carlson et al (2012) did furnish some insights into potential problems implementing and sustaining screening. 
Despite having the resources and focused attention of a funded clinical trial, these investigators lost a substantial proportion of their patients initially screened to follow-up, even when excluding from consideration those who were deceased. Furthermore, only a minority of distressed patients in either condition accessed services, with no group differences in outcome associated with group assignment, but those who accessed services improved more. This suggests the possibility that many of distressed patients are uninterested in services, despite encouragement for them to seek them, and that some wisdom of self-selection may determine which patients will seek services and benefit from them.

Screening is resource intensive, and questions can be raised as to what alternative purposes the resources consumed by screening could be put. These might include facilitating follow-up to ensure completion of referrals or dedication of the resources to addressing the large social disparities in completion of referrals. Simply put, apply the resources that would otherwise go to screening instead to facilitating completion of referrals for the minority of patients who want services, particularly those who are having low income or otherwise disadvantaged. Furthermore, because many services must be provided in the community rather than in the cancer centre, resources could be applied to ensuring the quality and intensity of these services, which cannot be presumed to be evidence-based or efficacious. Regardless, screening for distress should not be implemented without demonstration that it actually improves patient outcomes over routine care and that benefits exceed costs at patient and system levels. This criterion represents an application of the same standards that are applied to other screening in medical settings. Providing evidence of the benefits of screening takes precedence over any evidence of 'viability' or 'feasibility' in the absence of establishing efficacy.

\section{REFERENCES}

Carlson LE, Groff SL, Maciejewski O, Bultz BD (2010) Screening for distress in lung and breast cancer outpatients: a randomized controlled trial. J Clin Oncol 28: 4884-4891.

Carlson LE, Groff SL, Waller A, Bultz BD (2011) Clinical trial did not demonstrate benefits of screening patients with cancer for distress reply. J Clin Oncol 29: E279-E280.

Carlson LE, Waller A, Groff SL, Zhong L, Bultz BD (2012) Online screening for distress, the 6th vital sign, in newly diagnosed oncology outpatients: randomised controlled trial of computerised $v s$ personalised triage. $\mathrm{Br} \mathrm{J}$ Cancer 107: 617-625.

Raffle AE, Gray JEM (2007) Screening: Evidence and Practice. Oxford Press: NewYork, NY. 